\title{
An Evaluation of swine flu (Influenza A H3N2v)virus prediction using data mining and Conventional neural network techniques
}

\author{
Pilla Srinivas $^{1}$, Debnath Bhattacharyya ${ }^{2}$ and Divya Midhun Chakkaravarthy ${ }^{1}$ \\ ${ }^{1}$ Department of Computer Science and Multimedia, Lincoln University College, Malaysia \\ ${ }^{2}$ Department of Computer science and Engineering, K L Deemed to be University, KLEF, Guntur-522502, India
}

\begin{abstract}
Nowadays, The health care commercial enterprise collects huge amounts of healthcare data which, unfortunately, are not "mined" to discover hidden information. Data mining plays a significant role in predicting diseases. The database report of medical patient is not more efficient, currently we made an Endeavour to detect the most widely spread disease in all over the world named Swine flu. Swine flu is a respiratory disease which has Numeral number of tests must be requisite from the patient for detecting a disease. Advanced data mining techniques gives us help to remedy this situation. In this work we describes about a prototype using data mining techniques, namely Naive Bayes Classifier. The Data mining is an emerging research trend which helps in finding accurate solutions in many fields. This paper highlights the various data mining technique and Convolution Neural Network used for predicting swine flu diseases.
\end{abstract}

Keywords: Data Mining, Swine flu Disease, Naive Bayes Classifier and CNN

\section{INTRODUCTION}

Swine flu is one of the most infectious diseases which contain types of virus that causes thousands of deaths per year. Hence we have use a method of data mining to predict this disease using Naive Bayes classifier thus we can decrease the laboratory test cost as well as time also. "How can we turn useful data into information that can enable healthcare practitioners to make intelligent clinical decisions?" This is the main purpose for this work.

Swine flu is a respiratory disease caused by influenza viruses that infect the respiratory tract of pigs and result in a barking cough, decreased appetite, nasal secretions, and listless behavior the virus can be transmitted to humans. Swine flu viruses may mutate or change so that they are easily transmissible among humans.Swine influenza, is also called pig influenza or swine flu, hog flu and pig flu, is an infection caused by any one of several types of swine influenza viruses. Swine influenza virus (SIV) or swine origin influenza virus (S-OIV) is any strain of the influenza family of viruses that is endemic in pigs. In 2009, the known influenza include in strain and there are some subtypes of influenza A known as H1N1, H1N2, H2N1, H3N1, H3N2, and H2N3. Swine influenza virus is common throughout comes from pig populations.[1,2]

The virus transmit from pigs to humans is not common and does not always tend toward human flu, often resulting only in the productivity of antibodies in the blood of human. If transmission does cause human flu, it is known as zoonotic swine flu. People with regular come in contact to pigs are at increased risk of swine flu infection. Around the mid-20th century, the checking of influenza subtypes became possible, allowing exactly right diagnosis of transmission to humans. Since then, only 50 such transmissions have been confirmed. Swine flu is transmitted from person to person by inhalation or ingestion of droplets containing virus from people sneezing or coughing; it is not transmitted by eating cooked pork products. The newest swine flu virus that has caused swine flu is influenza A H3N2v. [7]

Some common symptoms of Swine flu are -

$\cdot$ Cough $\bullet$ Fever $\bullet$ Sore throat $\bullet$ Runny nose $\bullet$ Headache $\bullet$ Chill $\bullet$ Fatigue $\bullet$ Nausea.

\section{LITERATURE REVIEW}

Thakkar, Hasan and Desai were inspired to do this research by the study of mortality rate of swine flu[3]. This work focus on the aspect of medical diagnosis by learning predictions through the data collected for Swine flu. We proposed a method to identify swine flu by study 110 symptoms to decrease the cost incurred in the test of the disease. We developed prototype intelligent swine flu prediction software,usedNaive Bayes classifier technique for classifying the patients of swine flu. Based on the possibility of the diseases and guaranteed the accuracy of almost 63.3 percent. In August 2010, the World Health Organization declared the swine flu pandemic officially over. Cases of swine flu have been reported in India, with over 25000 positive test cases and 1370 deaths till March 2015[8]

\section{TECHNIQUES USED}

Concepts used are as follows: 


\section{A. Data Mining}

\section{B. Convolution Neural Networks}

\section{A. Data Mining}

- The cause of the 2009 swine flu was an influenza A virus type designated as H1N1. In 2011, a new swine flu virus was detected with new strain was named influenza A (H3N2) v. Only a few people (mainly children) were first infected, but officials from the U.S. Centers for Disease Control and Prevention (CDC) reported increased numbers of people infected in the 2012-2013 flu season. Currently, the swine flu virus kill more than 1500 people in India and it will rapidly spreading all over the world so recently there are not notice large numbers of people infected with H3N2v.Unfortunately, another virus termed H3N2 (note no "v" in its name) has been found and caused flu, but this strain is different from H3N2v. In general, all of the influenza A viruses have a structure similar to the $\mathrm{H} 1 \mathrm{~N} 1$ virus each type has a somewhat different $\mathrm{H}$ and/or $\mathrm{N}$ structure.

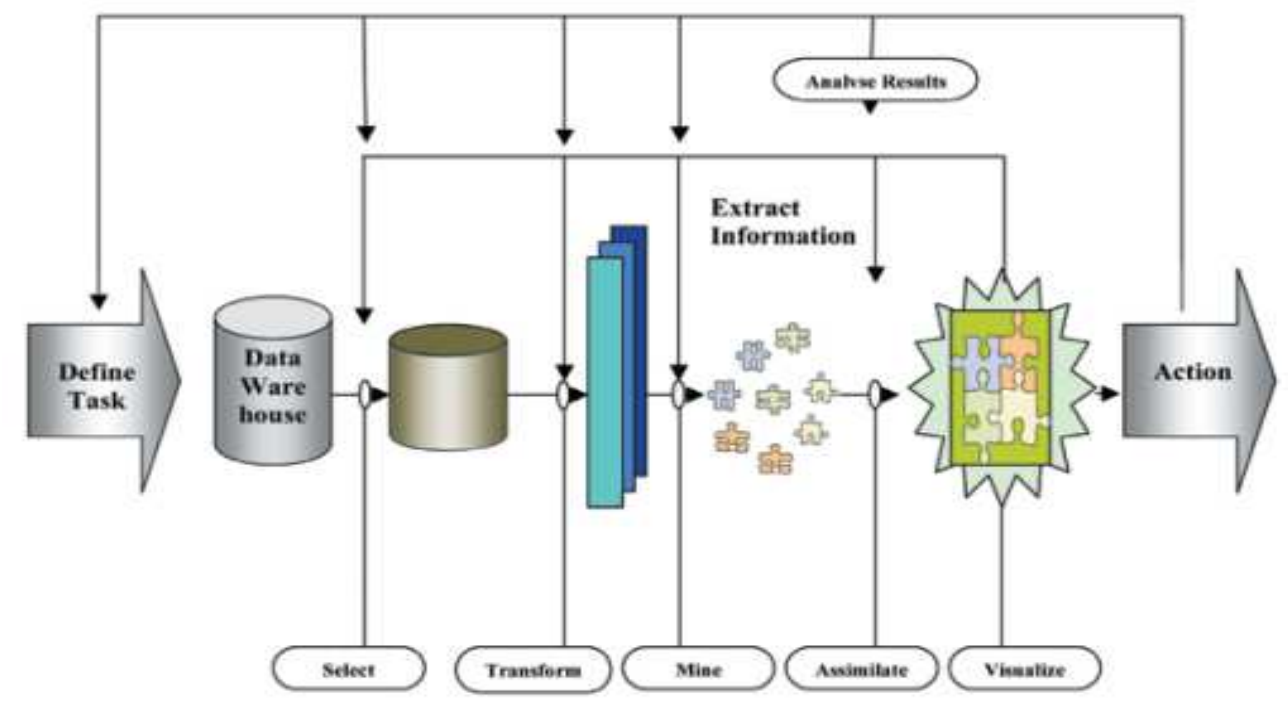

Fig.1: - Working of Data Mining

Technically, data mining is the process of finding correlations or patterns among different of fields in large relational databases. The step of the "Knowledge Discovery in Databases (KDD)" process, an interdisciplinary subfield of computer science, is the computational process of discovering patterns in large data sets involving methods at the intersection of artificial intelligence, machine learning, statistics, and database systems. The overall goal of the data mining process is to extract information from a data set and transform it into an understandable structure for further use.[10]

\section{B. Convolution Neural Networks}

- The Convolutional Neural Network (CNN) is a class of deep learning neural networks. CNNs represent a huge breakthrough in image recognition. They are most commonly used to analyze visual imagery and are frequently working behind the scenes in image classification.

\section{- A CNN has}

$\begin{array}{ll}\circ & \text { Convolutional layers } \\ \circ & \text { ReLU layers } \\ \circ & \text { Pooling layers } \\ \circ & \text { A Fully connected layer }\end{array}$

-A classic CNN architecture would look something like this:

Input $=>$ Convolution $=>$ ReLU $=>$ Convolution $=>$ ReLU $=>$ Pooling $=>\operatorname{ReLU}=>$ Convolution $=>\operatorname{ReLU}=>$ Pooling $=>$ Fully Connected

- A CNN convolves (not convolutes...) learned features with input data and uses 2D convolutional layers. This means that this type of network is ideal for processing 2D images. Compared to other image classification algorithms, CNNs actually use very little preprocessing. This means that they can learn the filters that have to be 
hand-made in other algorithms. CNNs can be used in tons of applications from image and video recognition, image classification, and recommender systems to natural language processing and medical image analysis.

- CNNs are inspired by biological processes. They're based on some cool research done by Hubel and Wiesel in the 60 s regarding vision in cats and monkeys. The pattern of connectivity in a CNN comes from their research regarding the organization of the visual cortex. In a mammal's eye, individual neurons respond to visual stimuli only in the receptive field, which is a restricted region. The receptive fields of different regions partially overlap so that the entire field of vision is covered.
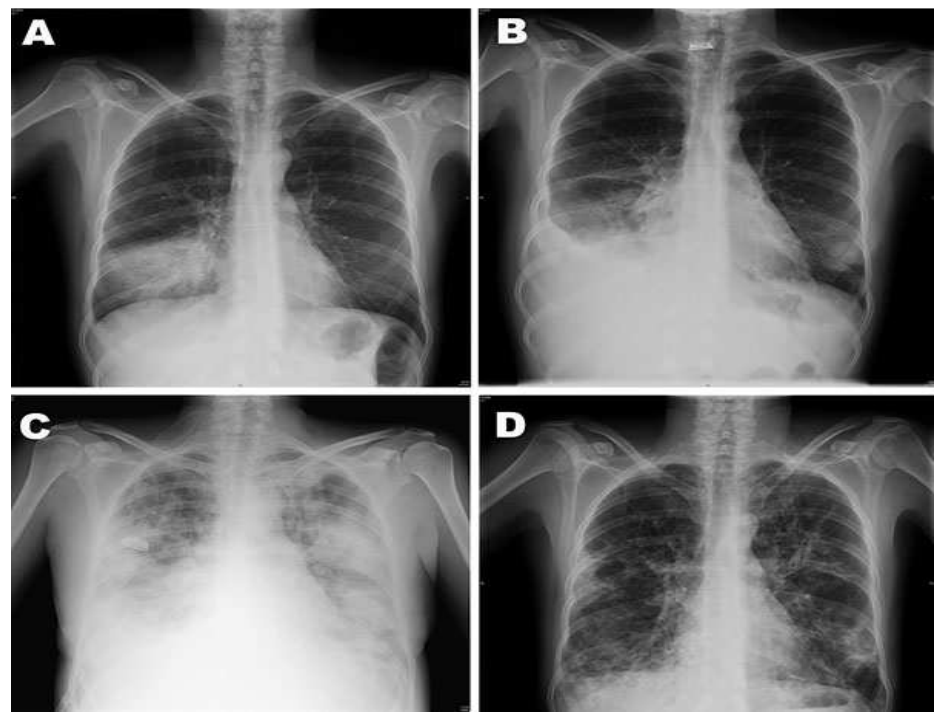

Fig. 2:- X-Ray image recognition

CNNs have an input layer, and output layer, and hidden layers. The hidden layers usually consist of convolutional layers, ReLU layers, pooling layers, and fully connected layers.

- Convolutional layers apply a convolution operation to the input. This passes the information on to the next layer.

- Pooling combines the outputs of clusters of neurons into a single neuron in the next layer.

- Fully connected layers connect every neuron in one layer to every neuron in the next layer.

In a convolutional layer, neurons only receive input from a subarea of the previous layer. In a fully connected layer, each neuron receives input from every element of the previous layer.

A CNN works by extracting features from images. This eliminates the need for manual feature extraction. The features are not trained. They are learned while the network trains on a set of images. This makes deep learning models extremely accurate for computer vision tasks. CNNs learn feature detection through tens or hundreds of hidden layers. Each layer increases the complexity of the learned features.

\section{$\mathrm{A} \mathrm{CNN}$ is}

-Starts with an input image

-Applies many different filters to it to create a feature map

-Applies a ReLU function to increase non-linearity

-Applies a pooling layer to each feature map

-Flattens the pooled images into one long vector.

Inputs vector into a fully connected artificial neural network. 
-Processes the features through the network. The final fully connected layer provides the "voting" of the classes that we're after.

-Trains through forward propagation and back propagation for many, many epochs. This repeats until we have a well-defined neural network with trained weights and feature detectors.

\section{A. Very Deep Convolutional Networks for Large-Scale Image Recognition (VGG-16)}

The VGG-16 is one of the most popular pre-trained models for image classification. Introduced in the famous ILSVRC 2014 Conference, it was and remains THE model to beat even today. Developed at the Visual Graphics Group at the University of Oxford, VGG-16 beat the then standard of AlexNet and was quickly adopted by researchers and the industry for their image Classification Tasks.

As you can see, the model is sequential in nature and uses lots of filters. At each stage, small $3 * 3$ filters are used to reduce the number of parameters all the hidden layers use the ReLU activation function. Even then, the number of parameters is 138 Billion - which makes it a slower and much larger model to train than others.

Additionally, there are variations of the VGG16 model, which are basically, improvements to it, like VGG19 (19 layers). You can find a detailed explanation

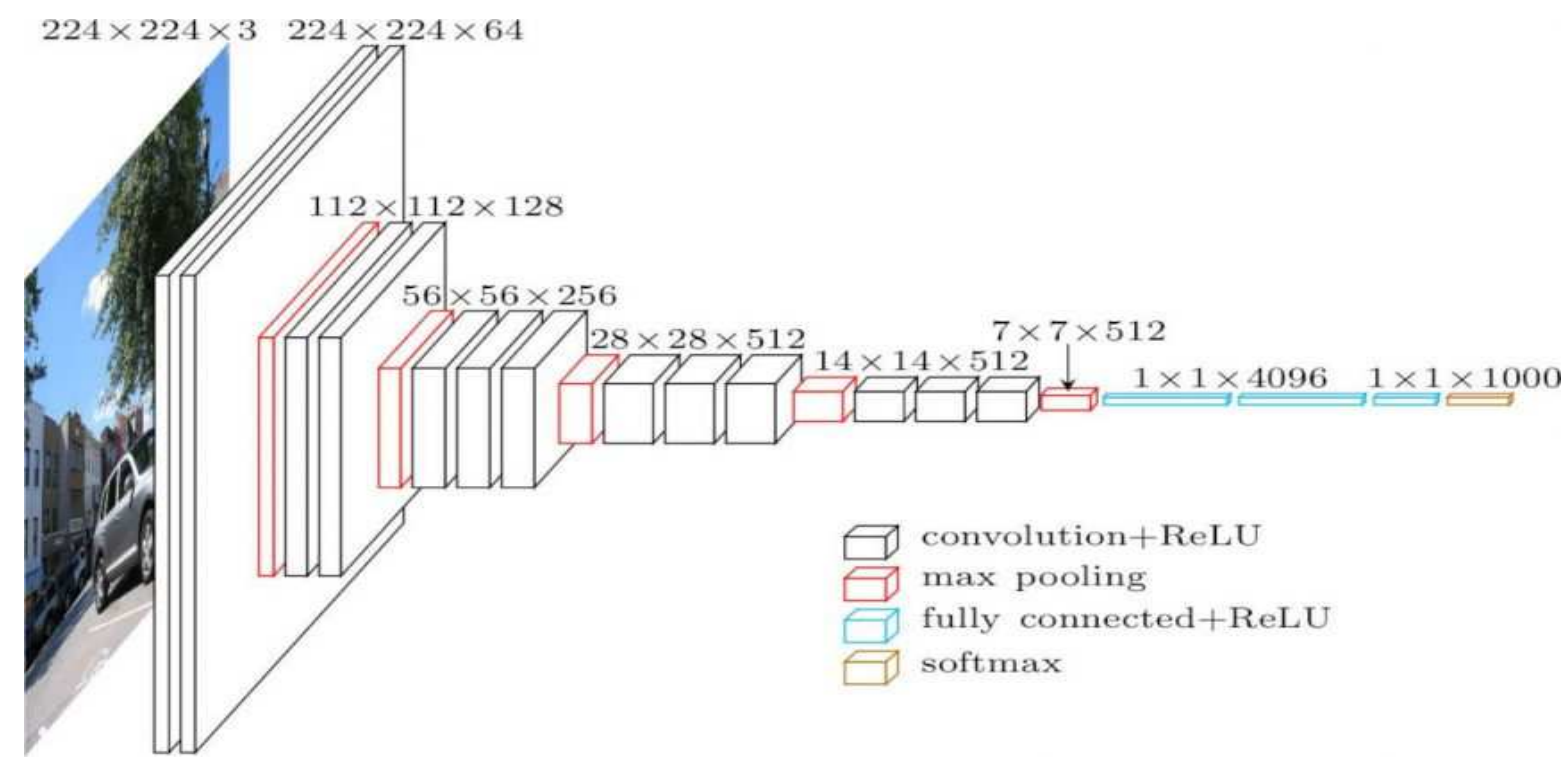

Fig.3:- Architectureof VGG-16 Model

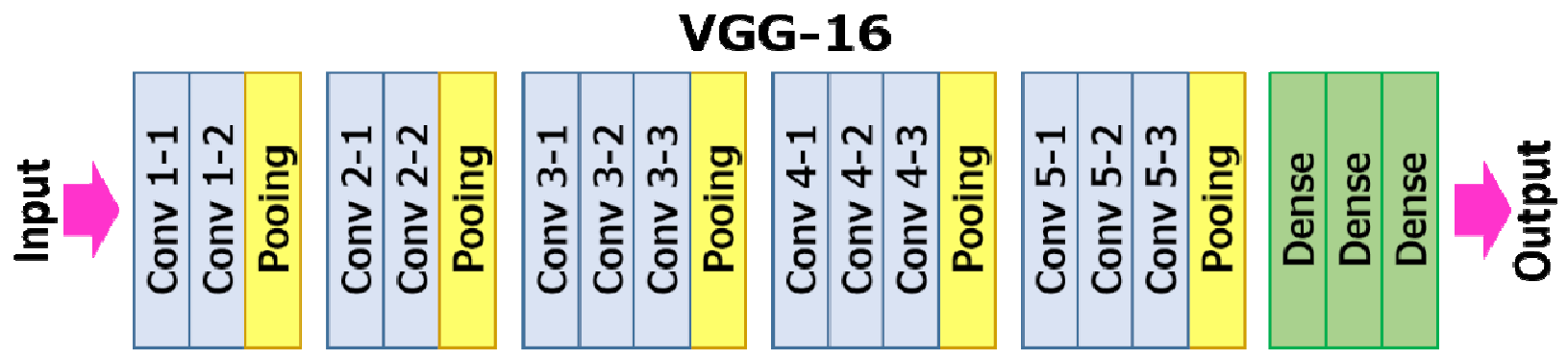

Fig.4:- Intuitive model of VGG-16 Model

The following are the layers of the model:

○ Convolutional Layers $=13$

○ Pooling Layers $=5$

- Dense Layers $=3$

Let us explore the layers in detail:

1.Input: Image of dimensions $(224,224,3)$.

\section{Convolution Layer Conv1:}


- Conv1-1: 64 filters

- Conv1-2: 64 filters and Max Pooling

○ Image dimensions: $(224,224)$

3. Convolution layer Conv2: Now, we increase the filters to 128

- Input Image dimensions: $(112,112)$

- Conv2-1: 128 filters

- Conv2-2: 128 filters and Max Pooling

4. Convolution Layer Conv3: Again, double the filters to 256, and now add another convolution layer

○ Input Image dimensions: $(56,56)$

- Conv3-1: 256 filters

○ Conv3-2: 256 filters

- Conv3-3: 256 filters and Max Pooling

5.Convolution Layer Conv4: Similar to Conv3, but now with 512 filters

○ Input Image dimensions: $(28,28)$

- Conv4-1: 512 filters

- Conv4-2: 512 filters

○ Conv4-3: 512 filters and Max Pooling

6. Convolution Layer Conv5: Same as Conv4

- Input Image dimensions: $(14,14)$

- Conv5-1: 512 filters

- Conv5-2: 512 filters

Conv5-3: 512 filters and Max Pooling

- The output dimensions here are $(7,7)$. At this point, we flatten the output of this layer to generate a feature vector

7.Fully Connected/Dense FC1: 4096 nodes, generating a feature vector of size (1, 4096)

8.Fully Connected /Dense FC2: 4096 nodes generating a feature vector of size (1, 4096)

9.Fully Connected /Dense FC3: 4096 nodes, generating 1000 channels for 1000 classes. This is then passed on to a Soft max activation function

\section{Output layer}

\section{B.Inception}

At only 7 million parameters, it was much smaller than the then prevalent models like VGG and AlexNet. Adding to it a lower error rate, you can see why it was a breakthrough model. Not only was this, but the major innovation in this work also another breakthrough - the Inception Module.

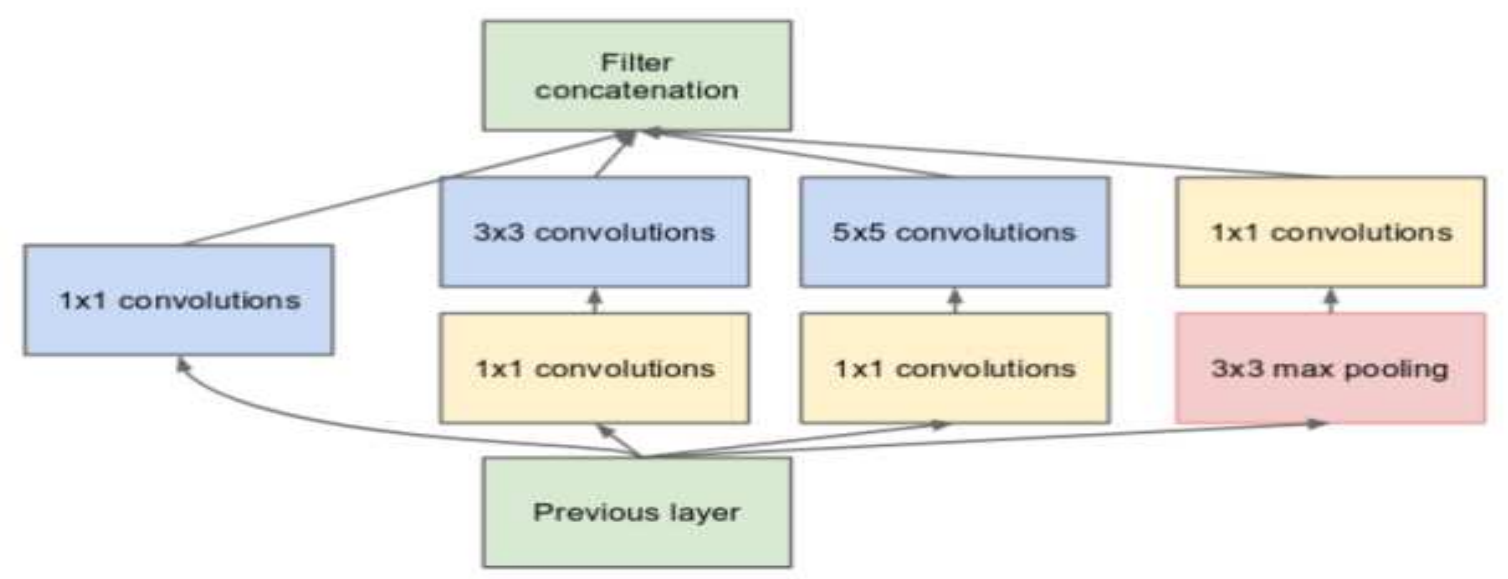

Fig5.: - Inception module with dimension reductions 
As can be seen, in simple terms, the Inception Module just performs convolutions with different filter sizes on the input, performs Max Pooling, and concatenates the result for the next Inception module. The introduction of the $1 * 1$ convolution operation reduces the parameters drastically.

\section{inception modules
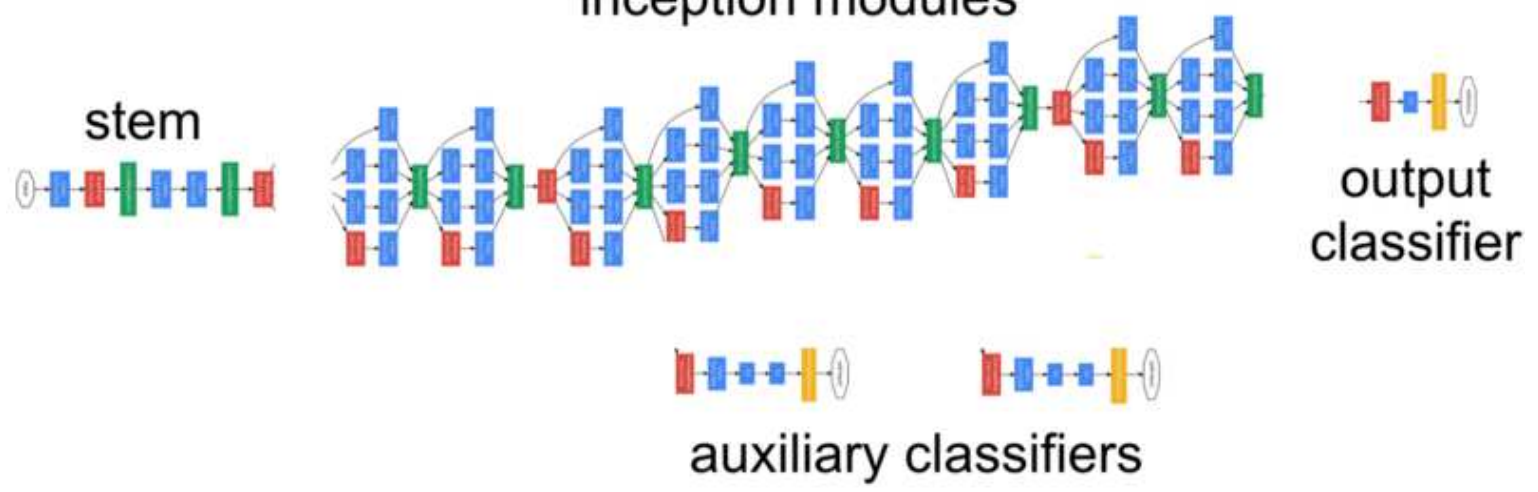

Fig.6:- InceptionV2 Model

The Inceptionv2 model was a major improvement on the Inceptionv1 model which increased the accuracy and further made the model less complex. In the same paper as Inceptionv2, the authors introduced the Inceptionv3 model with a few more improvements on v2.

The following are the major improvements included:

-Introduction of Batch Normalization

-More factorization

-RMSProp Optimizer

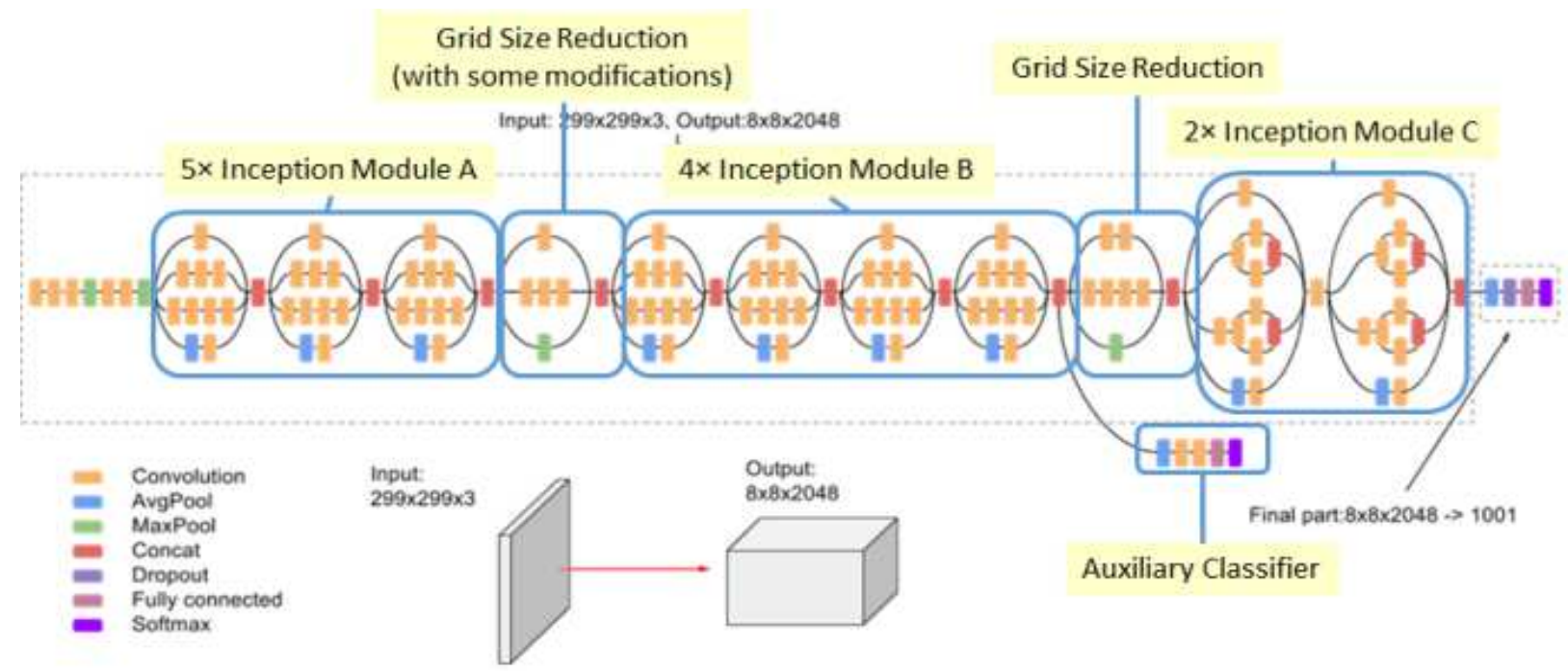

Fig.7. InceptionV3 Model

\section{C.ResNet50}

Just like Inceptionv3, ResNet50 is not the first model coming from the ResNet family. The original model was called the Residual net or ResNet and was another milestone in the CV domain back in 2015.

The main motivation behind this model was to avoid poor accuracy as the model went on to become deeper. Additionally, if you are familiar with Gradient Descent, you would have come across the Vanishing Gradient issue - the ResNet model aimed to tackle this issue as well. Here is the architecture of the earliest variant: ResNet34 (ResNet50 also follows a similar technique with just more layers) 


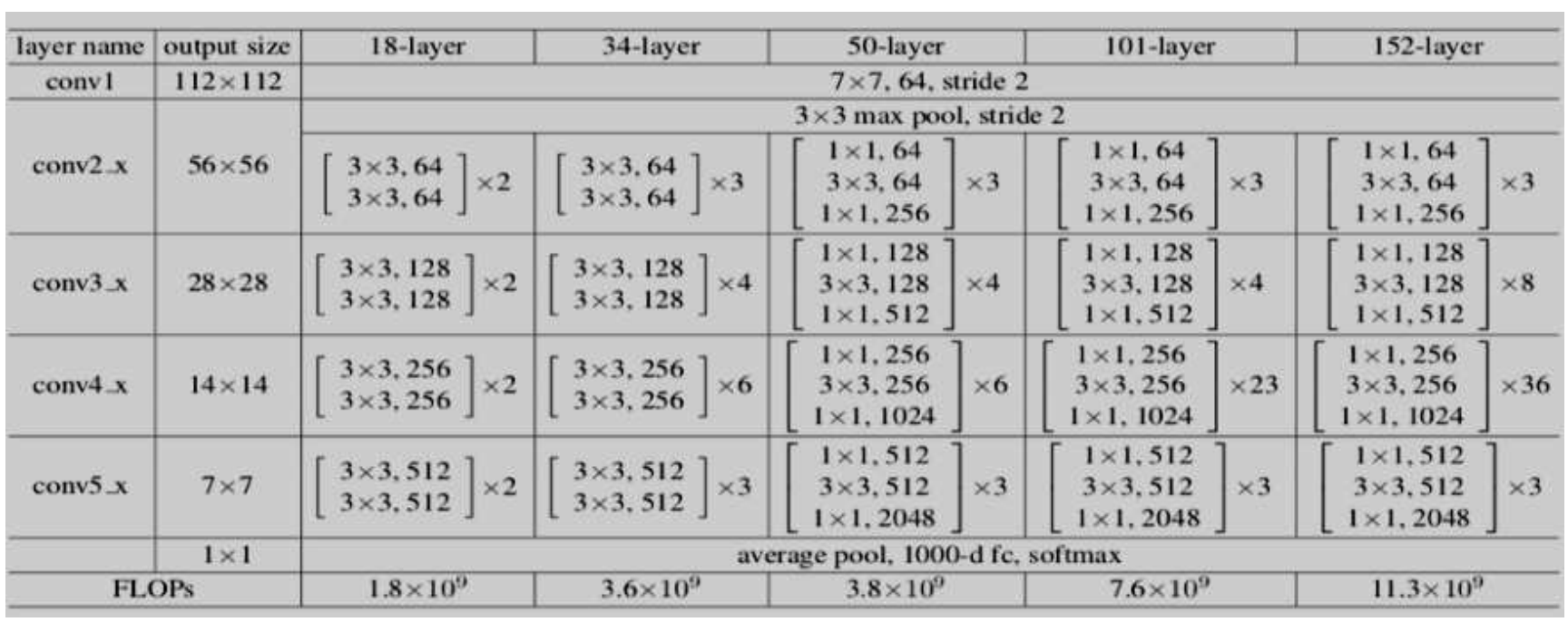

Fig.8: - Architecture of ResNet family in terms of layers

\section{EfficientNet}

We finally come to the latest model amongst these 4 that have caused waves in this domain and of course, it is from Google. In EfficientNet, the authors propose a new Scaling method called Compound Scaling. The long and short of it is this: The earlier models like ResNet follow the conventional approach of scaling the dimensions arbitrarily and by adding up more and more layers.

However, the paper proposes that if we scale the dimensions by a fixed amount at the same time and do so uniformly, we achieve much better performance. The scaling coefficients can be in fact decided by the user.

Though this scaling technique can be used for any CNN-based model, the authors started off with their own baseline model called EfficientNetB0

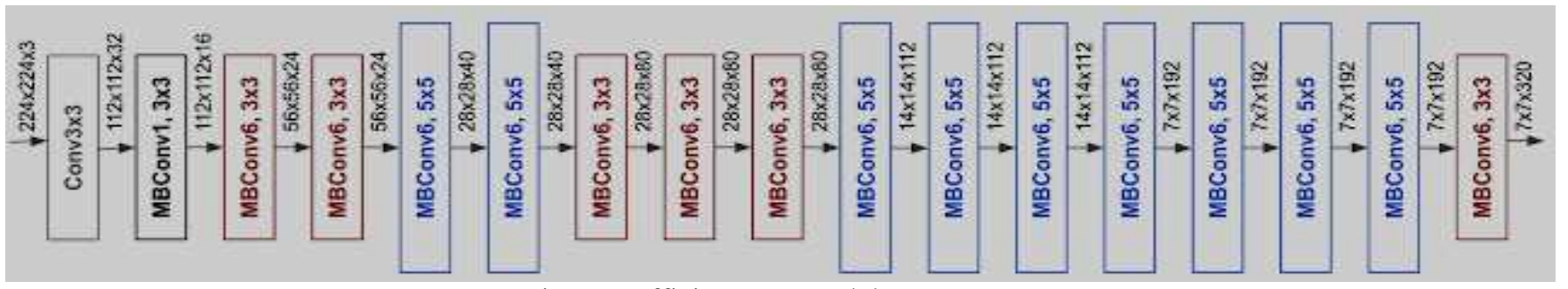

Fig.9 : - Efficient Net Model

MBConv stands for mobile inverted bottleneck Convolution (similar to MobileNetv2). They also propose the Compound Scaling formula with the following scaling coefficients:

-Depth $=1.20$

-Width $=1.10$

$\cdot$ Resolution $=1.15$

This formula is used to again build a family of Efficient Nets - EfficientNetB0 to EfficientNetB7.

The following is a simple graph showing the comparative performance of this family vis-a-vis other popular models: 


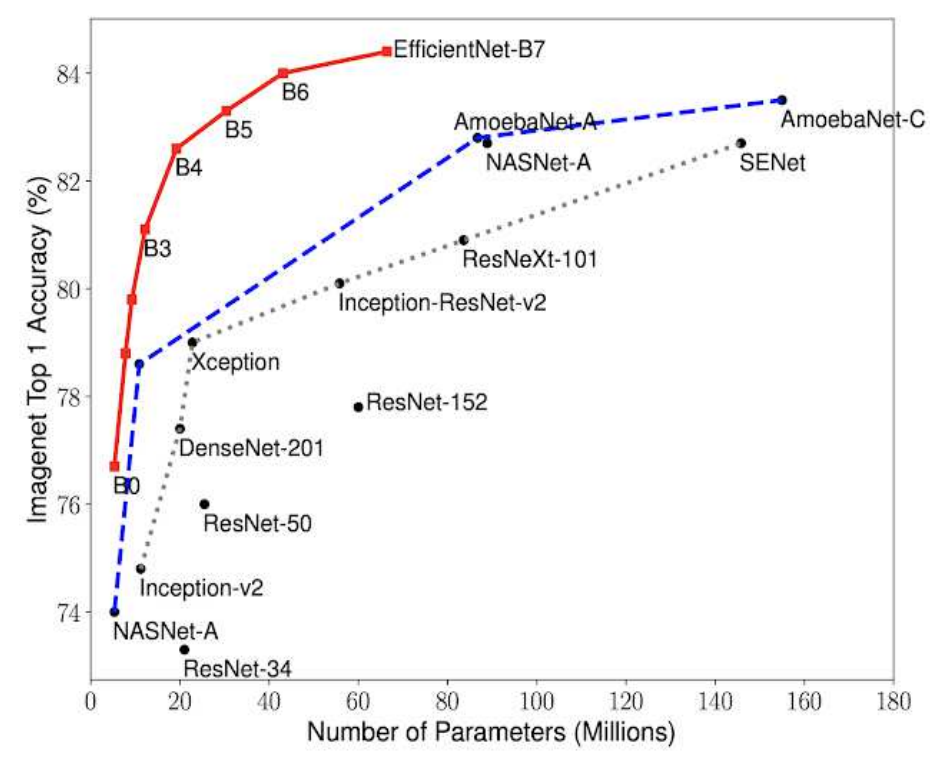

Fig.10:- Simple graph showing the comparativeperformance of this family vis-a-vis other popular models

\section{THEOREMS USED}

Here we use two types of theorems:

\section{A.Bayes Theorem}

\section{B.Naive Bayesian Classifier}

A. Bayes Theorem :Bayes Theorem is a simple mathematical formula used for calculating conditional probabilities.

Conditional probability is a measure of the probability of an event occurring given that another event has (by assumption, presumption, assertion, or evidence) occurred.

The formula isCV

$P(A / B)=\frac{P(B / A) \cdot P(A)}{P(B)}$

Which tells us: how often A happens given that $B$ happens, written $P(A \mid B)$ also called posterior probability, When we know: how often $B$ happens given that $A$ happens, written $P(B \mid A)$ and how likely $A$ is on its own, written $\mathrm{P}(\mathrm{A})$ and how likely $\mathrm{B}$ is on its own, written $\mathrm{P}(\mathrm{B})$.

In simpler terms, Bayes Theorem is a way of finding a probability when we know certain other probabilities.

\section{B. Naive Bayesian Classifier:}

Example: - Given all the patients we have seen their

\begin{tabular}{|l|l|l|l|l|}
\hline Chill & Runny nose & Headache & Fever & Swineflu \\
\hline Y & N & Mild & Y & N \\
\hline Y & Y & No & N & Y \\
\hline Y & N & Strong & Y & Y \\
\hline N & Y & Mild & Y & Y \\
\hline N & N & No & N & N \\
\hline N & Y & Strong & Y & Y \\
\hline N & Y & Strong & N & N \\
\hline Y & Y & Mild & Y & Y \\
\hline
\end{tabular}

Table1: Symptoms and Diagnosis are shown in the above table 
If there is a new entry of patient having following symptoms which listed below in Table no.2 So do we believe that a patient with following symptoms has the swine flu or not?

First, we compute all possible individual probabilities conditioned on the target attribute of Swine flu contained all probabilities of attribute of swine flu.

$\mathrm{P}($ swine flu $\mathrm{Y})=5 / 8=0.625$

$\mathrm{P}($ chills $=\mathrm{Y} \mid$ swine flu $=\mathrm{Y})=3 / 5=0.6$

$\mathrm{P}($ swine flu $=\mathrm{N})=3 / 8=0.375$

$\mathrm{P}($ chills $=\mathrm{Y} \mid$ swine flu $=\mathrm{N})=1 / 3=0.333$

Just like above we can simply compute the possible probabilities for all condition and this probabilities are enlist in Table no.3:- And then we decide that the $\mathrm{p}$ has split up into two cases one for $\mathrm{Y}$ and second for

$\mathrm{P} 1 \Rightarrow \operatorname{argmax} \mathrm{P}($ swine flu=Y) * $\mathrm{P}($ chills $=\mathrm{Y} \mid$ swine flu=Y) $* \mathrm{P}$ (runnynose=N $\mid$ swine flu=Y) * $\mathrm{P}$ (headache $=$ Mild $\mid$ swine flu $=\mathrm{Y}) * \mathrm{P}($ fever=N $\mid$ swine flu=Y)

$=0.625 * 0.6 * 0.2 * 0.4 * 0.2=0.006$

$\mathrm{P} 1=0.006$

$\mathrm{P} 2=>\operatorname{argmax} \mathrm{P}($ swine flu=Y) $* \mathrm{P}($ chills $=\mathrm{Y} \mid$ swine flu= $\mathrm{N}) * \mathrm{P}($ runnynose=N $\mid$ swine flu=N) * $\mathrm{P}$ (headache $=$ Mild $\mid$ swine flu $=\mathrm{N}) * \mathrm{P}($ fever $=\mathrm{N} \mid$ swine flu=N)

$=0.375 * 0.333 * 0.666 * 0.333 * 0.666$

$\mathrm{P} 2=0.0185$

Therefore, the argument of probability of P2 seems maximum than P1 so that patient not having the swine flu.

Types of Naive Bayes Classifier:

-Multinomial Naive Bayes: Feature vectors represent the frequencies with which certain events have been generated by a multinomial distribution. This is the event model typically used for document classification.

-Bernoulli Naive Bayes: In the multivariate Bernoulli event model, features are independent Booleans (binary variables) describing inputs. Like the multinomial model, this model is popular for document classification tasks, where binary term occurrence features are used rather than term frequencies.

-Gaussian Naive Bayes: In Gaussian Naive Bayes, continuous values associated with each feature are assumed to be distributed according to a Gaussian distribution (Normal distribution). When plotted, it gives a bell-shaped curve which is symmetric about the mean of the feature values.

The likelihood of the features is assumed to be Gaussian. Hence, conditional probability is given by:

$p(x)=\frac{1}{\sigma \sqrt{2 \pi}} e^{-\frac{1}{2}\left(\frac{x-\mu}{\sigma}\right)^{2}}$

Now, what if any feature contains numerical values instead of categories i.e. Gaussian distribution.

One option is to transform the numerical values to their categorical counterparts before creating their frequency tables. The other option, as shown above, could be using the distribution of the numerical variable to have a good guess of the frequency. For example, one common method is to assume normal or Gaussian distributions for numerical variables.

The probability density function for the normal distribution is defined by two parameters (mean and standard deviation).

$\mu=\frac{1}{n} \sum_{i=1}^{n} x_{i} \ldots \ldots \ldots .$. Mean 


$$
\begin{aligned}
& \sigma=\sqrt{\frac{1}{n-1}} \sum_{i=1}^{n}\left(x_{i}-\mu\right)^{2} \ldots \ldots . . . S t a n d a r d \text { Deviation } \\
& f(x)=\frac{1}{\sigma \sqrt{2 \pi}} e^{-\frac{1}{2}\left(\frac{x-\mu}{\sigma}\right)^{2}} \ldots \ldots . . . \text { Normal distribution }
\end{aligned}
$$

\section{Text Preprocessing:}

The next step is to preprocess text before splitting the dataset into a train and test set. The preprocessing steps involve Removing Numbers, Removing Punctuations in a string, Removing Stop Words, Stemming of Words and Lemmatization of Words.

\section{Constructing a Naive Bayes Classifier:}

Combine all the preprocessing techniques and create a dictionary of words and each word's count in training data.

1.Calculate probability for each word in a text and filter the words which have a probability less than threshold probability. Words with probability less than threshold probability are irrelevant.

2.Then for each word in the dictionary, create a probability of that word being in insincere questions and its probability insincere questions, then finding the conditional probability to use in naive Bayes classifier.

3.Prediction using conditional probabilities.

\section{V.CONCLUSION}

The Data mining technique can be used in collaboration with a naive bayes classifiers algorithm which used in diagnosing Swine flu disease. The proposed approach showed Promising results which may lead to further attempts to utilize information technology for diagnosing patients for Swine flu diseases. Here we used Naivebayes Classification rules which are easy to interpret. In future, we will try to get more the accuracy for the swine flu disease patient by increasing the various parameters suggested from the doctors by using different data mining techniques.

\section{REFERENCES}

[1]The Swine Influenza http://en.wikipedia.org/wiki/Swine_influenza

[2]The Swine Influenza http://www.medicinenet.com/swine_flu/article.htm

[3]Thakkar. Hasan and Desai, "Health care decision support system for swine flu prediction using Naivebayes classifier", International Conference on Advances in Recent Technologies in Communication and Computing.,IEEE,2010.

[4]www.tbevidence.org/documents/dxres/models/tb_diagnostics.pdf

[5]Swine flu paper http://www.ijarcsse.com/docs/papers/Volume_4/ 6_June2014/V4I6-0235.pdf

Mrs.G.Subbalakshmi, Mr.M.Chinna Rao "Decision Support in heart disease prediction system using Naivebays",IJCSE Indian journalof computer science and engineering, ISSN : 0976-5166 Vol.

[6]Mr. Pillasrinivas, Dr. Debnath Bhattacharyya and Dr. Divyamidhunchakkaravarthy "An artificial Intelligent based on system for Efficient Swine flupredictionusingNaive Bayesian classifier", IJCRR International Journal of Current Research Review, ISSN:0975-5241(online) Vol.12 . http://dx.doi.org/10.31781/IJCRR.2020.121519

[7]Machine Learning for Medical Diagnosis: History, State of the Art and Perspective by Igor Kononenko. [8]Hai Wang et. al.," Medical Knowledge Acquisition through Data Mining”, Proceedings of 2008 IEEE International Symposium on IT in Medicine and Education 978-1-4244-2511-2/.

[9]Kaur, H., Wasan, S. K.: "Empirical Study on Applications of Data Mining Techniques in Healthcare", Journal of Computer Science 2(2), 194-200, 2006.

[10]Health Care Decision Support System for Swine Flu Prediction Using Naive Bayes Classifier Artcom international conference, 978-1-4244-8093-7

[11]Data mining techniques, Available http://www.ibm.com/developerworks/opensource/library/ba-datamining techniques /index.html?ca=drs- , downloaded on 04 April 2013.

[12]Y P Munjal, Surendra K Sharm, API Textbook of Medicine, Ninth Edition, Two Volume Set, JP Medical Ltd, 2012, pp1156-1157.

[13]C. Chrysostomou, H. Partaourides and H. Seker, "Prediction of Influenza A virus infections in humans using an Artificial Neural Network learning approach," 2017 39th Annual International Conference of the IEEE Engineering in Medicine and Biology Society (EMBC), Seogwipo, 2017, pp.1186-1189.

[14]V. Rathod, U. Shaikh, O. P. Yadav and A. Rathore, "Swine Influenza Model Based Optimization using seasonal flu shots (SIMBO-SS)," 2015 4th International Conference on Reliability, Infocom Technologies and 
Optimization (ICRITO) (Trends and Future Directions), Noida, 2015, pp. 1-5.

[15]Incent AL, Ma W, Lager KM, Janke BH, Richt JA. Swineinfluenza viruses: a North American perspective. AdvVirusRes2008;72:127-54

[16]Gooskens J, Jonges M, Claas EC, Meijer A, Kroes AC. Pro-longed influenza virus infection during lymphocytopenia and frequent detection of drug-resistant viruses.J InfectDis2009;199:1435—41.

[17]Hompson WW, Shay DK, Weintraub E, Brammer L, CoxN, Anderson LJ, et al. Mortality associated with influenzaand respiratory syncytial virus in the United States. JAMA2003;289:179—86.

[18]Meijer A, Lackenby A, Hungnes O, Lina B, van-der-WerfS, Schweiger B, et al. Oseltamivir-resistant influenzavirus A (H1N1), Europe 2007—08 season. Emerg Infect Dis2009;15:552 —60.

[18] P.Rajendiran, K.R.Sekar, B.Sinivasan\&R.Manikandan, "Customer Relationship Management in the Manufacturing Industry using Data Mining Techniques", International Journal of Educational Science and Research (IJESR), Vol. 7, Issue 6, pp, 63-70

[19] Jaskaran Kaur \&ShevetaVashish, "Analysis of Different Clustering Techniques for Detecting Human Emotions Variation through Data Mining", International Journal of Computer Science Engineering and Information Technology Research (IJCSEITR), Vol. 3, Issue 2, pp, 27-36.

[20] Vishal Jain, Gagandeep Singh Narula\&Mayank Singh, "Implementation of Data Mining in Online Shopping System Using Tanagra Tool”, International Journal of Computer Science and Engineering (IJCSE), Vol. 2, Issue $1, \mathrm{pp}, 47-58$

[21] Wei-Hsuan Lee, Chien-Hua Wang, Jich-Yan Tsai \& Chin-Tzong Pang, "Association Rules Mining Combined with FAHP”, International Journal of Computer Science and Engineering (IJCSE), Vol. 5, Issue 2, Feb - pp, 33-44

[22] Sunil Yadav\&Gajanand Sharma, "Improvisation of Data Mining Techniques in Cancer Site among Various Patients Using Market Basket Analysis Algorithm", BEST: International Journal of Management, Information Technology and Engineering (BEST: IJMITE), Vol. 3, Issue 10, pp, 137-144

[23] Rajendran L, "Bird Flu Viruses Avian Influenza during 2000-2019: A Time Sequence Analysis on Cab Databases". IMPACT: International Journal of Research in Humanities, Arts and Literature (IMPACT: IJRHAL), Vol. 8, Issue 10, pp, 11-20 